\title{
Ciberconducta y dependencia emocional en parejas jóvenes
}

\author{
Eduardo Espinar, Izabela Zych* y Antonio J. Rodríguez-Hidalgo \\ Universidad de Córdoba, España
}

(Recibido 14 Febrero, 2015; Aceptado 27 Febrero, 2015)

RESUMEN: El uso de las TIC y de los cibermedios está llevando a una nueva forma de socialización y comunicación entre los jóvenes. El medio virtual se ha convertido en un nuevo escenario para relacionarse en el que también surgen y se mantienen relaciones de pareja. A su vez, las relaciones entre las parejas jóvenes pueden ser muy satisfactorias, aunque varios estudios también han descrito la existencia de diversos problemas tales como algunas conductas inadecuadas hacia la pareja o la existencia de la llamada dependencia emocional. Por ello, en el presente trabajo se describe el uso de diferentes cibermedios entre los jóvenes y su relación con los niveles de dependencia emocional. Primero, se aplicó un cuestionario sobre el uso de los cibermedios, propio y percibido en la pareja. Conjuntamente, se aplicó el test IRIDS-100 de dependencia emocional. Ambos instrumentos fueron respondidos por 100 estudiantes de edades comprendidas entre 19 y 32 años matriculados en la Universidad de Córdoba. Los resultados han mostrado un uso elevado, tanto propio como percibido en las parejas, de WhatsApp, Facebook, Twitter y Youtube. Igualmente, se ha encontrado la existencia de correlaciones positivas entre el uso propio y percibido en la pareja de Tuenti y Youtube y la dependencia emocional. Dado que los cibermedios han cambiado la forma de relacionarse de los jóvenes, con sus iguales y con sus parejas, los datos de este trabajo contribuyen al estudio de este tema, proponiendo a su vez nuevas líneas de investigación relacionadas con la dependencia emocional expresada a través de la ciberconducta.

Palabras clave: parejas jóvenes, TIC, dependencia emocional, ciberconducta.

\section{Cyber-behavior and emotional dependency in young couples}

\begin{abstract}
The use of the ICT and the cyber media is leading to new forms of socialization and communication among youth. Virtual environment has become a new context for relating to other people which also includes the establishment and development of couple relationships. At the same time, couple relationships in youth can be very satisfactory, although many studies also described the existence of different problems such as inadequate behaviors towards the partners or emotional dependency. Thus, the current work describes the use of different cyber media among youth and its relationship to the levels of emotional dependency. First, a questionnaire on the use of cyber media in oneself and perceived in a partner was answered by the participants. At the same time, the IRIDS-100 questionnaire for the assessment of the emotional dependency was responded. Both instruments were answered by 100 students between 19 and 32 years old,


enrolled in the University of Córdoba. Results showed frequent use of WhatsApp, Facebook, Twitter and Youtube in oneself and also perceived in the partner. Moreover, positive correlations were found between its use in oneself and perceived in the partner and emotional dependency. Taking into account that the cyber media have changed the way of relating among young people, with their peers and their couples, the results of the current study contribute to this topic suggesting at the same time new lines of studies related to the emotional dependency expressed through the cyber media.

Key words: young couples, ICT, emotional dependency, cyber-behavior.

\section{INTRODUCCIÓN}

La sociedad actual, se ve asediada por continuos cambios tecnológicos, sociales, intelectuales, económicos, etc. Dichos cambios parecen producirse a gran velocidad, influyendo en las personas y sus relaciones sociales. Es más, tanto las costumbres como las sensaciones habituales en el pasado no muy lejano, parecen haberse modificado (Méndez y Rodríguez, 2011). En este sentido, uno de los grandes cambios más acusados en nuestra sociedad postindustrial contemporánea es el avance y nueva necesidad de las tecnologías de la información y comunicación (TIC).

En muchas ocasiones, el objetivo principal perseguido a la hora de diseñar algunas de las TIC, ha sido en facilitar las actividades y tareas de los usuarios (Corral y Echeburúa, 2010). No obstante, algunos trabajos sobre las mismas demuestran que puede producirse justo el efecto contrario, es decir, que ciertas actividades resulten más complicadas por el hecho de llevarlas a cabo con el uso de las TIC (Gil, Feliu, Rivero y Gil, 2003). Esta situación ocurre principalmente, cuando en determinadas situaciones, dichas herramientas se convierten en un fin y no en un medio. La adquisición de un nuevo teléfono móvil con determinadas prestaciones, actualizado a su última versión, puede llegar a ser un objetivo principal para muchos jóvenes. A su vez, la creación de un perfil en las redes sociales a través de las TIC puede dominar la vida de un adolescente al facilitar la existencia de una nueva identidad "digital" que le resulte más beneficiosa, distorsionando incluso el mundo real (Echeburúa y De Corral, 2010).

Tal y como se ha comentado anteriormente, el uso de las TIC, junto con las ventajas e inconvenientes que conlleva, está cambiando la sociedad moderna. Dentro de éstas, se ha atribuido mucha importancia a la aparición y el uso del Internet, con un aumento de más de 2700 millones de usuarios en el mundo (Fundación Orange, 2013). Tal es la propagación de dicha comunicación en referencia a las TIC, que el $38 \%$ de la población mundial es cibernauta, siendo China el lugar donde se concentra más de la quinta parte de uso (Fundación Orange, 2013).

En los últimos años, se han producido cambios que han facilitado aun más el acceso a éste. Por ejemplo, seis de cada diez cibernautas se conectan a través de dispositivos móviles, que pueden tener instaladas todas las redes sociales, apli- 
caciones y programas necesarios para la nueva ciber-comunicación, o comunicación digital, siendo el teléfono móvil el más destacado por ser un punto referente para la conexión de Internet y comunicación a nivel mundial. En referencia a lo anterior, según el último estudio del Instituto Nacional de Estadística (INE) en el año 2013 en España, el 94,2\% de la población total utiliza el teléfono móvil diariamente, y un 98,2\% (la cifra más alta), son personas jóvenes de 16 a 24 años. En el apartado de haber utilizado Internet al menos una vez en semana en los últimos tres meses, de nuevo el sector de 16 a 24 años supera al resto de población con un $94,4 \%$ de respuestas afirmativas. A nivel regional, concretamente en Andalucía, ha habido un incremento destacado en cuanto a los cibernautas que acceden a la red desde el dispositivo móvil, colocándose por encima de la media nacional (Fundación Orange, 2013).

A su vez, hay que recordar que Internet comenzó como fuente de información y ha acabado siendo fuente de interacción social (Flores, 2009). Prueba de ello se concentra en el gran uso de los cibernautas en redes sociales como Facebook con más de 1000 millones de usuarios, o Twitter con 200 millones, en detrimento de las redes sociales locales de cada país (Ivars, 2013). Según lo revisado en la literatura científica, sobre el uso de redes sociales en universitarios Andaluces (Gómez, Farias y Roses, 2012), un 91,2\% de los participantes de dicho estudio, se reconoce como usuario de alguna red social, siendo Facebook, Twitter y Tuenti, las redes más utilizadas por dicha población. Sin embargo, Fotolog, Netlog, Myspace, Flickr, Hi5 y Xing ocuparían los últimos puestos de uso en la citada población. Las razones o causas que determinan dicho uso en los jóvenes, vienen motivadas por factores de autoidentidad, confianza en sí mismos, compensación social y entorno social (Colás, González y de Pablos, 2013). Además de tener un uso principalmente marcado por un carácter social, suponen un medio de comunicación sencillo, accesible y rápido sin suponer una barrera la distancia física o temporal. En dichas redes se pueden compartir ideas, emociones, noticias, tareas, enlaces a otras páginas o contenidos, archivos de todo tipo, etc. Resurgiendo una nueva identidad (virtual), enlazada a estos cibermedios, gestionando un perfil según el fin al que vayamos a utilizar dicha red social, delimitando cada código de comunicación (manera de expresarse) y cada lista de contactos a dicho fin, lo que supondría establecer diferentes "roles virtuales" sin dejar de ser en ningún momento la misma persona (Caldevilla, 2010).

El portal web "Youtube" tiene una mención especial en distinción a las demás redes sociales, o cibermedios. Dicho portal web es utilizado por 13,5 millones de usuarios al mes en España, que representaría el 54,25\% de población total de Internet en el país (Lavado, 2013). Youtube se distingue de las redes sociales en que es una web cuyo fin se basa en el carácter audiovisual de la misma, permitiendo a los usuarios "subir", descargar, o comentar vídeos a través de un nombre de usuario y contraseña. Youtube es líder empresarial en visualización de videos online, y su éxito radica en la cuantificación de los videos "más vistos" entre sus millones de usuarios, ya sea de carácter televisivo, o de carácter particular. Éste medio obtuvo el premio al "invento del año" en 2006, por la revista "Time" 
(Murolo, 2010). Al igual que Youtube, la aplicación Whatsapp tiene un apartado distinto al de las redes sociales, ya que se trata de una aplicación de chat o mensajería instantánea que nació en 2009, y que permite el envío entre sus contactos de fotos, vídeos y archivos de todo tipo, se usa mayoritaria y principalmente a través del teléfono móvil (Hidalgo, 2013).

Con todo ello, Internet se ha convertido en un importante escenario de socialización entre personas jóvenes, no siendo utilizado únicamente como fuente y contexto de información como ya se viene comentando anteriormente sino, ambién se utiliza como medio para compartir experiencias y comunicarse (Del Rey, Ortega Ruiz y Sánchez, 2012). Parece que la población joven, utiliza las redes sociales o cibermedios de comunicación principalmente para satisfacer sus necesidades comunicativas, establecer una comunicación de manera instantánea, sencilla y ociosa, además de suponer para ellos la construcción de su ser social, llegando a pensar muchas de las personas jóvenes encuestada encuestados, que es imprescindible disponer una red social propia o interactuar a través de la mensajería instantánea para disfrutar de una vida social plena (Bernal y Angulo, 2012; Espinar Ruiz y González Ríos, 2009).Teniendo en cuenta este contexto, no parece sorprendente que Internet se haya convertido también en un lugar en el que las personas jóvenes viven sus relaciones de pareja. Dichas relaciones de pareja se basan principalmente en la comunicación, tanto para las parejas que se forman en la red como en las parejas formadas en persona y se mantienen a través de Internet (Moral, 2001). Las parejas crean su propio lenguaje codificado para entenderse, y el erotismo de la relación se traslada en una fantasía subjetiva (Sotomayor, 2008).

Por todo lo descrito anteriormente, uno de los objetivos de este trabajo es analizar el uso propio y la percepción de uso que se tiene sobre la pareja (actual o pasada) referente a redes sociales y mensajería instantánea, en las pesonas jóvenes universitarios de la ciudad de Córdoba. Con ello, se pretende comprobar si tal como citan en los diferentes artículos sobre las TIC, las redes sociales y diferentes cibermedios de comunicación, se están convirtiendo en una nueva forma de socialización entre los jóvenes, siendo así en este caso, si en las parejas universitarias está emergiendo una nueva forma de "virtualizar" la afectividad tal como lo denominan Alfama, Bona y Callén (2005). Según estos autores, las TIC digitalizan los afectos disminuyendo la proximidad temporal, y consiguen codificar a un lenguaje alfanumérico los sentidos, sustituyendo los olores, el tacto, las miradas, en definitiva "lo real" por algo más potenciador que el simple texto de una carta. Las TIC consiguen darle un nuevo sentido y una nueva dirección a las emociones debido a los nuevos y cada vez más completos y complejos dispositivos electrónicos, sumado a los factores; tiempo, espacio y número de usuarios receptores afectados.

En este contexto, cabe preguntarse si los problemas que presentan las parejas en las interacciones cara a cara tienen relación con su interacción en el ciberespacio. Uno de esos problemas es la llamada dependencia emocional, que se define como los comportamientos desadaptativos adictivos hacia otra persona, 
resultando una asimetría de rol en la relación (Moral y Sirvent, 2009). Jorge Castelló (2010), la define como un "patrón persistente de necesidades emocionales insatisfechas que se intentan cubrir desadaptativamente con otras personas".

El dependiente emocional no presenta sus síntomas únicamente con su pareja, ya que puede presentarlos estando sin ella, aunque dicha situación resulta extraña ya que siempre está en búsqueda de disponer de una relación sentimental (Castelló, 2010). Por ello, cabe destacar que dicha necesidad no es subyacente o equiparable a una relación de "amor utópico". Para poder distinguirla, se define el "amor utópico" como aquel amor que para quien lo experimenta debe ser una decisión conjunta o bilateral, y resulta ventajosa y enriquecedora, uniendo pasión intimidad compromiso, además de disponer de libertad propia de ambas partes (García, 2013). Por ello se debe recalcar lasdiferencias existentes entre ambas, y distinguir esa necesidad extrema afectiva que tienen los dependientes emocionales, que le hacen necesitar e intentar cubrir unas necesidades emocionales y de apoyo, incluido en aquellas situaciones que la persona es capaz de autosatisfacerse por sí mismo (Gómez-Acosta y Izquierdo Martínez, 2013).

Las personas dependientes tienden a la exclusividad de las relaciones sentimentales, suelen necesitar constantemente la total aprobación por parte de los demás, idealizan a sus parejas, y pueden escoger a personas ególatras, narcisistas y seguras de sí mismas (Moral y Sirvent, 2009; Rodríguez, 2013). Estas personas suelen presentar una baja autoestima, y se caracterizan por tener pánico ante la posibilidad de la ruptura de la relación. A su vez, manifiestan cierto déficit en sus relaciones sociales, y el miedo a la soledad y a la excesiva necesidad de continuar con su pareja les hace permitir y soportar conductas y actitudes inadaptativas e intolerables por parte de ésta (Castelló, 2010).

Considerando la población sujeta a este estudio, líneas de investigación similares resaltan que las personas jóvenes consideran la entrega al otro como uno de los factores más importantes en una relación, asumiendo que a la vez de placentero puede llegar a ser doloroso, lo que puede suponer riesgo de llegar a una dependencia (Pradas Cañete y Perles Novas, 2012).

Dado que las TIC suponen un nuevo medio de comunicación para las personas de la sociedad postindustrial moderna y un nuevo medio de interacción entre las parejas, existe la posibilidad de que su uso esté relacionado con la dependencia emocional. Este trabajo se centrará en estudiar el uso de los denominados cibermedios de comunicación (Tuenti, Facebook, Twitter, Youtube, etc en jóvenes universitarios) y la percepción que tienen del uso por parte de las parejas en las relaciones sentimentales, (actuales o pasadas). Igualmente, se estudiará la dependencia emocional en los y las jóvenes universitarios y por último la relación entre el uso y la percepción del uso de estos medios por parte de las parejas de los cibermedios de comunicación y la dependencia emocional. Teniendo en cuenta la revisión de la literatura, se plantea que el uso propio de los cibermedios tales como Youtube, Facebook, Twitter y Tuenti, es muy frecuente por parte de las personas jóvenes universitarios participantes de este estudio (hipótesis 1); al ser una muestra de población general, las medias muestran valores bajos de 
dependencia emocional entre los participantes de este estudio respecto a sus parejas (hipótesis 2) pero existiendo una alta relación positiva entre el uso de los cibermedios y la presencia de dependencia sentimental entre los participantes de este estudio (hipótesis 3).

\section{MÉTODO}

\section{Participantes}

El presente trabajo contó con la participación de 100 sujetos matriculados en la titulación del segundo ciclo de psicopedagogía de la facultad Ciencias de la Educación de la Universidad de Córdoba. Las edades de los participantes estaban comprendidas entre 19 y 32 años con una media de 22,48 (DT=2,82), resultando de dichos 100 participantes, 86 mujeres y 14 hombres (86\% mujeres, $14 \%$ hombres).

\section{Diseño y Procedimiento}

Se trata de un estudio trasversal del tipo ex post facto realizado mediante encuesta (Montero \& León, 2007). La aplicación del cuestionario se hizo durante sus horas de clase habituales. El profesorado previamente informado, dejó media hora de su clase correspondiente indistintamente del turno al que correspondiera (mañana y tarde) para que el alumnado pudieran rellenar el cuestionario dentro de cada aula. En el apartado de instrucciones del mismo, se especificaba que todos los datos que iban a cumplimentar eran totalmente confidenciales y por tanto, tratados de manera anónima.

\section{Instrumentos}

Para conocer el uso de los cibermedios por parte de los jóvenes universitarios y la percepción que tienen de uso sobre sus parejas, se elaboró un Cuestionario sobre el Uso de los Cibermedios. Éste consistió en 17 preguntas sobre el uso de diferentes cibermedios. se solicitaba a los participantes que indicaran la frecuencia de uso de los cibermedios presentados en el test, y a su vez, que indicaran la percepción de uso que creian que podian tener sus parejas de 1 (Nunca) a 5 (A cada hora).

Para medir la dependencia emocional se administró el Inventario de Relaciones Interpersonales y Dependencias Sentimentales (IRIDS-100; Sirvent y Moral, 2005). Dicho cuestionario está compuesto por 100 ítems con afirmaciones sobre comportamientos referidos a las relaciones de pareja actuales o pasadas. Las afirmaciones se presentan en el verbo presente y el sujeto debe responder en torno a lo que ha sido habitual en su vida, aunque ahora hayan cambiado dichas situaciones o comportamientos. Los ítems se responden en una escala tipo Likert valorado de 1 (muy en desacuerdo) a 5 (muy de acuerdo). El cuestionario pre- 
senta buenas propiedades psicométricas con un alfa de Cronbach de 0,96 para la puntuación total en el presente estudio.Para este estudio, se han utilizado las siguientes subescalas:

- La subescala de dependencia afectiva, mide un apego emocional intenso dirigido hacia una persona con demandas afectivas reiteradas. Dicha subescala se compone de 24 ítems y varía de un valor mínimo de 24 a un valor máximo de 120 . Presenta un alfa de Cronbach de 0,89 por lo que resulta tener una elevada fiabilidad.

- La subescala de codependencia, mide un tipo de conducta sobreprotectora y de cuidados, caracterizándose por un excesivo vigilo, girando toda su atención en la otra persona, llegando a descuidarse a sí mismo. Dicha subescala se compone de 25 ítems y varía de un valor mínimo de 25 a un valor máximo de 125 . Presenta un indicador de alfa de Cronbach de 0,86 por lo que resulta tener una elevada fiabilidad.

- La subescala de bidependencia, se caracteriza por conductas de atracción y obsesión intensas hacia el otro, sensación de inescapabilidad (sentirse atrapado en una relación sentimental), sin ninguna autonomía, delegando todas las decisiones en la otra persona. Dicha subescala se compone de 26 ítems y varía de un valor mínimo de 26 a un valor máximo de 130 . Presenta un indicador de alfa de Cronbach de 0,88 por lo que presenta una elevada fiabilidad.

\section{RESULTADOS}

Tal como se indica en la Tabla 1, la herramienta de comunicación más utilizada tanto en lo asumido por los sujetos; $(M=4,68, D T=0,98)$, como en lo percibido sobre sus parejas actuales o pasadas; $(M=4,53, D T=1,14)$, es la aplicación de mensajería instantánea WhatsApp. En la autoevaluación de uso de las herramientas utilizadas, le sigue el servicio de correo electrónico Hotmail; ( $M=3,87$ $D T=1,05)$. Con escasa diferencia respecto a Hotmail, se encuentra el portal de videos de Youtube; $M=3,73$, $(D T=0,91)$. Por último destacar Facebook, como la red social más utilizada por los jóvenes, $(M=3,31, D T=1,47)$. Por el contrario, la red social Netlog sería para los jóvenes universitarios la menos utilizada; $(M=$ $1(D T=0)$. 
Tabla 1. Medias y Desviaciones Típicas de las Frecuencias de Usos de Diferentes Herramientas de Comunicación por Parte de los Participantes y la Percepción de Uso en sus Parejas

\begin{tabular}{lcc} 
& $\begin{array}{c}\text { ¿Qué medios sueles } \\
\text { utilizar? } \\
M(D T)\end{array}$ & $\begin{array}{c}\text { iQué medios suele utilizar } \\
\text { tu pareja? (actual o } \\
\text { pasada) } \\
M(D T)\end{array}$ \\
\hline WhatsApp & $4,68(0,98)$ & $4,53(1,14)$ \\
Line & $2,10(1,21)$ & $2,11(1,32)$ \\
Skype & $1,75(1,07)$ & $1,82(1,11)$ \\
Msn & $1,30(0,68)$ & $1,31(0,76)$ \\
Facebook & $3,31(1,47)$ & $3,05(1,51)$ \\
Tuenti & $3,23(1,20)$ & $2,96(1,33)$ \\
Badoo & $1,07(0,49)$ & $1,11(0,51)$ \\
Youtube & $3,73(0,91)$ & $3,42(1,39)$ \\
Flickr & $1,11(0,49)$ & $1,16(0,64)$ \\
Twitter & $3,23(1,84)$ & $2,73(1,73)$ \\
Myspace & $1,02(0,20)$ & $1,05(0,32)$ \\
Fotolog & $1(0)$ & $1,05(0,26)$ \\
Netlog & $1(0)$ & $1,04(0,31)$ \\
Instagram & $1,59(1,19)$ & $1,49(1,12)$ \\
Gmail & $2,79(1,47)$ & $2,12(1,35)$ \\
Yahoo & $1,16(0,59)$ & $1,18(0,59)$ \\
Hotmail & $3,87(1,05)$ & $2,98(1,39)$ \\
\hline
\end{tabular}

En cuanto a la percepción que tienen los jóvenes sobre el uso de las herramientas de comunicación en sus parejas actuales o pasadas, se presenta, como ya se ha mencionado, WhatsApp como líder indiscutible de herramienta de comunicación entre ellos. Según lo percibido por los sujetos, siguiendo a WhatsApp, el portal de videos Youtube sería el más utilizado en sus parejas; $(M=3.42$, $D T=1.39)$. La red social Facebook también lidera, en las creencias de uso, sobre las demás, $(M=3.05, D T=1.51)$. Y cómo red social percibida por los jóvenes por las menos usadas por sus parejas también se encontró Netlog con sus propios criterios. $(M=1,04 ; D T=0,31)$.

Respecto a las diferentes subescalas de la escala de dependencia emocional aplicada en el estudio, (Sirvent y Moral, 2005) (dependencia afectiva, codependencia, bidependencia y total) los alfas de Cronbach, las medias y desviaciones típicas de cada subescala se pueden apreciar en la tabla 2. 
Tabla 2. Medias y Desviaciones Típicas de las Diferentes Subescalas de la Dependencia Emocional

$M(D T)$

Dependencia afectiva

Alfa de Cronbach $=0,89$

Codependencia

Alfa de Cronbach $=0,86$

Bidependencia

Alfa de Cronbach $=0,88$

TOTAL DEPENDENCIA

Alfa de Cronbach $=0,96$
$49,21(13,54)$

$46,69(12,32)$

$190,68(42,78)$

La subescala dependencia afectiva se compone de 24 ítems, y varía de 24 a 120. Como se puede apreciar en la tabla 3, la media en los participantes del presente estudio es de 49,21 (DT=13,54).

La subescala codependencia se compone de 25 ítems, y varía de 25 a 125. Como se puede apreciar en la tabla 3, la media de los participantes del presente estudio es de 55,17 (DT=2,85).

La subescala bidependencia se compone de 26 ítems, y varía de 26 a 130. Tal como aparece en la tabla 3, la media de los participantes es de 46,69 (DT=12,32).

Los resultados sugieren niveles bajos de dependencia emocional en los participantes. La media de cada subescala se corresponde a los valores de respuesta "muy en desacuerdo" o "en desacuerdo" con respecto a la presencia de la misma.

Teniendo en cuenta las relaciones entre las diferentes variables de la dependencia emocional medida por el test IRIDS con diferentes subescalas y el uso de diferentes herramientas de comunicación a través de Internet, considerando las tres subescalas y el total, se han buscado relaciones significativas (véase tablas

Tabla 3. Relación entre el Uso de Cibermedios y la Dependencia Emocional

Tuenti

Youtube

\begin{tabular}{lll} 
Dependencia afectiva & $0,295 * *$ & $0,221 *$ \\
Codependencia & 0,185 & $0,325^{* *}$ \\
Bidependencia & $0,266^{*}$ & $0,248^{*}$ \\
Total & $0,271^{*}$ & $0,29 * *$ \\
\hline$*_{p}<.05, * * p<.01, * * * p<.001$ &
\end{tabular}


Tabla 4. Relación entre el Uso de Cibermedios Percibido en la Pareja y las Diferentes Subescalas de Dependencia Emocional

\begin{tabular}{lll}
\hline & Tuenti & Youtube \\
Dependencia afectiva & $0,488^{* *}$ & $0,245^{*}$ \\
Codependencia & $0,41 * *$ & $0,217^{*}$ \\
Bidependencia & $0,487^{* *}$ & 0,187 \\
Total & $0,476^{* * *}$ & 0,176 \\
\hline
\end{tabular}

$* p<.05, * * p<.01, * * * p<.001$

3 y 4). En este sentido, se han encontrado correlaciones significativas entre la utilización de la red social Tuenti (yo) y las subescalas; dependencia emocional $(r=0,295 ; p \leq 0,001)$ bidependencia $(r=0,266 ; p \leq 0,05)$ y con el total del cuestionario $(r=0,271 ; p \leq 0,01)$. En este sentido, también se han encontrado correlaciones positivas significativas entre la utilización de Youtube (yo) y las tres subescalas de la dependencia emocional y con el total de las mismas; dependencia afectiva y Youtube $(r=0,22, p \leq 0,05)$; codependencia y Youtube $(r=$ $0,325 ; p \leq 0,01)$ bidependencia y Youtube $(r=0,248 ; \mathrm{p} \leq 0,05)$ y total y Youtube $(r=0,29 ; p \leq 0,01)$

En cuanto a la correlación entre la percepción del uso de diferentes medios por parte de la pareja actual o pasada y la dependencia (véase tabla 4) se han encontrado fuertes correlaciones positivas entre el uso de la red social Tuenti con dependencia afectiva $(r=0,488 ; p \leq 0,01)$, codependencia $(r=0,41 ; p \leq 0,01)$, bidependencia $(r=0,421 ; p \leq 0,01)$, y total $(r=0,476 ; p \leq 0,01)$. Resultando a su vez, la percepción de uso del portal de videos de Youtube de la pareja y dos de las diferentes subescalas del test IRIDS; correlación positiva entre la dependencia afectiva y Youtube $(r=0,245 ; p \leq 0,05)$, y codependencia y Youtube $(r=0,217$; $p \leq 0,05)$.

No se han encontrado correlación significativa en la utilización de los demás cibermedios, y las diferentes subescalas.

\section{DISCUSIÓN}

Según lo descrito en la introducción de este estudio, las personas jóvenes de la sociedad actual presentan nuevas maneras de sociabilizarse y comunicarse, ya no únicamente de manera directa y personal, sino que también están emergiendo nuevas formas de comunicarse y relacionarse a través de las TIC en el ciberespacio o espacio virtual (Caldevilla, 2010). Según estudios que se centran en esta cuestión, casi la totalidad de los jóvenes encuestados usan redes sociales (Gómez et al., 2012). Por ello, el primer objetivo de este estudio ha sido en cono- 
cer los cibermedios jóvenes universitarios encuestados de la ciudad de Córdoba. De acuerdo a los resultados obtenidos, se observa que el uso de la aplicación de mensajería instantánea WhatsApp es el cibermedio o herramienta más utilizada entre los jóvenes y también la que se percibe como más utilizada por parte de sus parejas. Teniendo en cuenta su fácil instalación en un dispositivo móvil, y el elevado porcentaje que utiliza el teléfono móvil en España (94,2\% de la población total española) y en concreto los jóvenes (98,2\% de entre 16 y 24 años) cifras extraídas del INE (2013), no es de extrañar que la mayor parte de los jóvenes lo usen para y con diferentes fines (educativo, familiar, social, laboral, etc.) siendo el principal objetivo el de comunicarse y socializarse (Espinar Ruiz y González Ríos, 2009). En este sentido, se puede sugerir que el elevado uso (a cada hora) de dicha aplicación entre los jóvenes de la población universitaria, puede resultar en tanto beneficioso respecto al ahorro de tiempo que supondría en realizar diversas gestiones, como por ejemplo, no teniendo que llamar a diferentes personas por separado para dar un mismo mensaje, pudiendo crear un grupo de conversación dentro de la aplicación. Sin embargo, no todo resulta ser beneficioso, puede tratarse de un fenómeno perjudicial si se realiza un uso abusivo y no responsable del mismo.. En este sentido, la realización de las actividades cotidianas de los jóvenes, de carácter educativo, laboral o social, podría verse afectada y limitada por un uso excesivo de las TIC.., peligro del que ya han alertado otros autores (Echeburúa y De Corral, 2010).

Aunque tal como se acaba de exponer, la aplicación Whatsapp ocupa una gran parte del tiempo de los y las jóvenes universitarios en su uso, no es excluyente, ni el único cibermedio que dicha población utiliza. Los resultados de este trabajo indican que las redes sociales más utilizadas a cada hora son; Twitter y Facebook seguida de Tuenti respecto al uso propio. Igualmente la frecuencia coincide en la percepción de uso en la pareja. Éstas tres redes sociales, coinciden en diversos estudios (Colás et al., 2013; Flores, 2009; Gómez et al., 2011; Monge Benito y Olabarri Fernández, 2011) como los cibermedios más utilizados en comparación con las demás plataformas o redes sociales, aunque bien es cierto, que cada una se asigna a una población y a un rango de edad específicos.

En general, Twitter y Facebook son redes sociales muy utilizadas con 1000 millones de usuarios en Facebook y 200 millones de usuarios en Twitter a nivel mundial (Ivars, 2013). Por lo que la conexión y comunicación con usuarios de todo el mundo resulta más posible en detrimento de las redes sociales de cada país. A su vez, tanto Facebook como Twitter tienen una imagen más formal para los universitarios y con un público con el que comunicarse más dispar en referencia a la edad y la proximidad temporal. Cabe añadir que el uso de Facebook es mayor frente a la otra red social, en este caso, Tuenti. Esto podría deberse a la percepción por parte de los jóvenes universitarios como más completa respecto a la última, ya que Facebook dispone de la integración de aplicaciones por parte de desarrolladores de software externos, además de poseer más funciones que Tuenti (grupos de discusión, eventos, pestañas en los perfiles, publicidad, entre otras) (Monge y Olabarri, 2011). Aun así, es importante también resaltar que el 
acceso a Tuenti depende de una invitación por correo electrónico de un usuario ya registrado, mientras que en Facebook, su registro es directo y voluntario, lo que implicaría que la red Tuenti está compuesta por un grupo de usuarios que gira en torno al mismo rango de edad, mientras que en Facebook existiría un rango más amplio (Monge y Olabarri, 2011)

Cabe destacar también el cibermedio "Youtube", merece una mención especial las redes sociales por tener una función bien distinta, siendo lo más visitado entre los participantes de este estudio después de Twitter, tanto en el uso propio como en el uso percibido de los participantes en sus parejas. No hay que olvidar que Youtube es un portal web dónde se pueden compartir vídeos creados por los propios cibernautas, considerándose como un elemento complementario a la televisión, radicando su éxito en su carácter informativo, psicológico y formativo. A su vez, es utilizada como cibermedio o enlace para publicitar las grandes empresas sus marcas comerciales (Lavado, 2013). Por tanto, se confirma la primera hipótesis existiendo un elevado uso de determinados cibermedios por parte de los y las jóvenes de este estudio, tal como venían ya evidenciando otros autores (Colás et al., 2013; Flores, 2009; Gómez et al., 2012, etc).

Teniendo en cuenta que la sociedad actual se encuentra relación con en continuo cambio y evolución como ya se ha citado y referenciado a las TIC y al uso de Internet, también en dicha sociedad, surgen nuevas dependencias que implican una gran relevancia socio-sanitaria cómo la dependencia emocinal (Moral y Sirvent, 2009). Teniendo en cuenta esto, y que los y las jóvenes tienen la creencia de que la entrega hacia la otra persona es uno de los principales factores de una relación, asumiendo que puede resultar igual de placentero que doloroso (Pradas et al., 2012), se ha pretendido conocer en este estudio dependencia emocional en sus relaciones de pareja. En este sentido, según los resultados obtenidos, se ha demostrado que los niveles de dependencia emocional en los participantes han sido bajos.

Cómo ya se citó en la introducción, las TIC se han convertido en un nuevo contexto de relaciones interpersonales entre los jóvenes (Ortega et al., 2012). Teniendo en cuenta también la importancia de la comunicación emocional en la red (Belli, Harré y Íñiguez, 2009), se ha pretendido analizar la relación entre el uso de los cibermedios y la dependencia emocional. Los resultados en este sentido indican que existe una fuerte relación, tanto en el uso personal como en el uso percibido sobre las parejas, de la red social Tuenti y el portal web Youtube, con las diferentes subescalas de la dependencia sentimental. Por lo que a medida que aumenta el uso de los cibermedios Youtube y Tuenti tanto en el propio uso, como en la creencia de uso sobre sus parejas, aumenta la dependencia emocional. Respecto a la relación con Youtube, puede ser debido a que el perfil de dependiente emocional corresponde con una persona que "idealiza" su relación de pareja según Castelló, (2005), pudiendo provocar conductas que giran en torno a fomentar dichos síntomas. En este caso Youtube potencia la "atadura" del usuario, ya que permite el encadenamiento de vídeos afines o similares buscados por éste, (en el caso del dependiente emocional, vídeos afines a la idealización de la relación 
de pareja), consiguiendo permanecer delante de la pantalla o dispositivo móvil durante horas, y resultando un fin de puro entretenimiento más que de comunicación (Murolo, 2013). En cuanto a Tuenti, según el estudio de Monge y Olabarri (2011), es la red social utilizada, mayoritaria y principalmente por los más jóvenes, considerada por los participantes de dicho estudio como una red social para comunicarse entre los más amigos y conocidos al ser su registro a través de invitación, como ya se ha citado anteriormente. Esto potencia la comunicación entre los más allegados y resulta tener la barrera añadida respecto a Facebook, de no poder contactar con otras personas que no sean del entorno próximo, por lo que coincidiría con algunas de las características de las personas dependientes emocionales como la exclusividad de las relaciones interpersonales (Castelló, 2005), al tener controlado ese aspecto en dicha red social. También podría coincidir con la característica de la constante aprobación por parte de su entorno, (Castelló, 2005) al poder "subir" y por tanto publicar fotos de su relación en la red social, dónde se muestre aparente "felicidad" o comentarios en el "tablón" de su pareja de manera reiterada, demandando conductas "virtuales de afecto". Según los resultados obtenidos en este sentido y en relación a la tercera y última hipótesis planteada en esta investigación, se evidencia que ésta se confirma al existir relación entre diferentes cibermedios (Tuenti y Youtube) y la dependencia emocional.

Teniendo en cuenta los resultados de este trabajo que demuestran una relación significativa entre el uso de los cibermedios y la dependencia emocional, cabe preguntarse si existe algún tipo de dependencia emocional expresada de manera específica en el ciberespacio. En este sentido, se podría plantear, por ejemplo, que las personas dependientes de sus parejas en relaciones cara a cara también muestren esa dependencia a través de la ciberconducta. Sería recomendable que los estudios futuros profundizaran en esta línea de trabajo.

\section{REFERENCIAS}

Alfama E., Bona, Y., y Callén, B. (2005). La virtualización de la afectividad. AtheneaDigital. Revista de pensamiento e investigación Social,7, 1-17.

Belli, S., Harré, R., y Iñiguez, L. (2009). Emociones en la Tecnociencia: La Performance de la Velocidad. Revista de Ciencias Sociales Prisma Social, 3,141.

Bernal, C. y Angulo, F. (2013). Interacciones de los jóvenes andaluces en las redes sociales. Comunicar, 20, 25-30.

Caldevilla Domínguez, D. (2010). Las Redes Sociales. Tipología, uso y consumo de las redes 2.0 en la sociedad digital actual. Documentación de las Ciencias de la Información, 33, 45-68.

Castello, J. (2005). Dependencia emocional. Características y Tratamiento. Barcelona: Psicología Alianza Editorial.

Colás, P., González, T., y de Pablos, J. (2013). Juventud y redes sociales: Motivaciones y usos preferentes. Comunicar, 20, 15-23 
Echeburúa, E. y De Corral, P. (2010). Adicción a las nuevas tecnologías y a las redes sociales en jóvenes: un nuevo reto. Adicciones, 22, 91-96.

Espinar Ruíz, E. y González Río, M.J. (2009). Jóvenes en las redes sociales virtuales: un análisis exploratorio de las diferencias de género. Feminismo/s: Revista del Centro de Estudios sobre la Mujer, 14, 87-106. España(2013). Informe anual sobre el desarrollo de la sociedad de la información, 2013. Madrid. Fundación Orange. Recuperado de http://www.proyectosfundacionorange.es/docs/eEspana_2013_web.pdf.

Flores Vivar, J.M. (2009). Nuevos modelos de comunicación, perfiles y tendencias en las redes sociales. Comunicar, 33, 73-81.

Gil, A., Feliu, J., Rivero, I. y Gil, E. P. (2003). ¿Nuevas tecnologías de la información y la comunicación o nuevas tecnologías de relación? Niños, jóvenes y cultura digital(artículo en línea). UOC. (Fecha de consulta: 25/09/2013). Recuperado de http://www.uoc.edu/dt/20347/index.html.

Gómez, M., Roses, S.,y Farias, P. (2012). El uso académico de las redes sociales en universitarios. Comunicar, 19, 131-138.

García Faet, B. (2013). El "amor-funámbulo": contradicciones político-emocionales de las nuevas relaciones amorosas. Revista Latinoamericana de Estudios sobre Cuerpos, Emociones y Sociedad, 2, 23-36.

Hidalgo Rodríguez, G. M. (2013). Uso del teléfono móvil: Posibilidades Didácticas Y Riesgos en los Jóvenes. (Trabajo Fin de Master). Universidad de Almería, Almería.

Instituto Nacional de Estadística (2013). Equipamiento y uso de TIC en los hogares. Cifras INE (www.ine.es).

Ivars Medina, J. (2013). Empresa 2.0. Oltra Gutiérrez, J.V. (Proyecto final de carrera). Escuela Técnica superior de Ingeniería Informática. Universidad de Valencia. (Artículo en línea) (Fecha de consulta: 5/11/2013) Recuperado de: http://riunet.upv.es/bitstream/handle/10251/27557/Memoria.pdf?sequence=1

Izquierdo Martínez, S. A. y Gómez Acosta, A. (2013). Dependencia afectiva: abordaje desde una perspectiva contextual. Psychologia: avances de la disciplina, 7,81-91.

Lavado, A. (2013). El consumo de YouTube en España. Global Media Journal México, 7, 76-92.

Monge Benito, S. y Olabarri Fernández, M. E. (2011). Los alumnos de la UPV/ EHU frente a Tuenti y Facebook: usos y percepciones. Revista Latina de comunicación social, 66, 79-100.

Montero, I.,y León, O. G. (2007). A guide for naming research studies in Psychology. International Journal of Clinical and Health Psychology, 7, 847862.

Moral, F. (2001). Aspectos psicosociales de la comunicación y de las relaciones personales en Internet. Anuario de psicología, 32, 13-30.

Moral, M. V. y Sirvent, C. (2007). Codependencia y género: análisis explorativo de las diferencias en los factores sintomáticos del TDS- 100. Analesde Psiquiatría, 23, 90-91. 
Moral, M. V. y Sirvent, C. (2009). Dependencia Afectiva y Género: Perfil Sintomático Diferencial en Dependientes Afectivos Españoles. Revista Interamericana de Psicología/Interamerican Journal of Psychology, 43, 230-240.

Murolo, N.L. (2010). Post Zapping: Transmitetúmismo. Youtube como la televisión postmoderna. Razón y palabra 72. Campus tecnológico de Monterrey. (Artículo en línea) (Fecha de consulta: 29/09/2013). Recuperado de:Http:// www.razonypalabra.org.mx/N/N71/VARIA/14\%20MUROLO-REVISADO. pdf.

Ortega, R., del Rey, R. y Sánchez, V. (2012). Nuevas Dimensiones de la Convivencia Escolar y Juvenil. Ciberconducta y Relaciones en la red: Ciberconvivencia. (NIPO: 030-12-244-7). Universidad de Córdoba: España Secretaría General Técnica.

Pradas Cañete, E., y Perles Novas, F. (2012). Resolución de conflictos de pareja en adolescentes, sexismo y dependencia emocional. Quaderns de psicologia. International Journal of Psychology, 14, 45-60.

Rodríguez de Medina Quevedo, I. (2013). La Dependencia Emocional En Las Relaciones Interpersonales. Revista electrónica de investigación Docencia Creativa, 2,143-148.

Sirvent, C.y Moral, M.V. (2005). Test de Dependencias Sentimentales TDS-100. Oviedo, España: Fundación Instituto Spiral.

Sirvent, C. y Moral, M.V. (2007).Presentación del TDS-100: Test de Dependencias Sentimentales de Sirvent y Moral. Anales de Psiquiatría 23, 94-95.

Sotomayor García, G.E. (2008). Atrapados en la red del am@r: Un análisis del Lenguaje y las Emociones en la CMO. (Artículo de reflexión). Revista Educación, Comunicar, Tecnología, 4. 